\title{
PATHOLOGY/BIOLOGY
}

\author{
Amanda O. Fisher-Hubbard, ${ }^{1}$ M.D.; LokMan Sung, ${ }^{1,2}$ M.D.; Sean A. Hubbard, ${ }^{3}$ M.S., Ph.D.; and \\ Leigh Hlavaty, ${ }^{1,2}$ M.D.
}

\section{Hyperthermia, Thermal Injuries, and Death from a Forced Convection Heat Source: A Case Report and Experimental Model}

\begin{abstract}
Heat-related deaths of children are most often encountered in the context of enclosed vehicles in summer months. Deviating from this, a 16-month-old boy was found unresponsive in a stroller that was placed adjacent to a space heater during mid-winter. The cause of death was hyperthermia and thermal injuries. Manner of death determination was difficult due to alleged surrounding circumstances. To understand the time-course of this child's injuries, a child death scene investigation was performed; the stroller and space heater were recovered. In a re-enactment of the events, a slaughtered pig approximating the child's size was warmed using a water bath and placed in the stroller beside the space heater. Cutaneous temperature measurements showed rapid initial temperature rise with subsequent steady increases. Tanning of the skin was seen on periodic direct observations. Internal temperature monitoring illustrated steady increases. This experiment was essential in classifying the manner of death as homicide.
\end{abstract}

KEYWORDS: forensic science, hyperthermia, thermal injury, burns, pediatric, neglect, homicide

A National Association of Medical Examiners position paper specifies heat-related deaths as those caused or contributed to by high ambient temperatures. This diagnosis is rendered from the circumstances surrounding the death, investigative reports concerning environmental temperature, and/or measured antemortem body temperatures (1).

Heat-related illnesses and syndromes lie on a continuum ranging from mild to severe $(2,3)$. The most benign of these illnesses is heat rash, a type of skin irritation that results from excessive perspiration (2). Heat edema and heat syncope result from the vasodilation that occurs as the body tries to compensate for elevated temperatures (3). Heat cramps are painful muscle spasms that occur during heavy exercise and are thought to be the result of dilutional hyponatremia as an individual attempts to rehydrate with free water. Heat exhaustion, the most common heat-related illness, is characterized by volume depletion that occurs in heat stress. It is classically divided into water depletion and salt depletion forms, although most individuals present with a combination of the two. These patients have nonspecific systemic complaints and profuse sweating, usually with a core body temperature of less than $40^{\circ} \mathrm{C}$. They will not have signs of severe central nervous system damage (3). Heat stroke is a life-threatening condition that occurs when the heat-dissipating mechanisms of the body are unable to compensate for the increased heat (4). It may occur

\footnotetext{
${ }^{1}$ Department of Pathology, University of Michigan, 1301 Catherine Street, Ann Arbor, MI 48109.

${ }^{2}$ Wayne County Medical Examiner's Office, 1300 E. Warren Avenue, Detroit, MI 48207.

${ }^{3}$ NES Technologies, Inc., P.O. Box 2308, Champaign, IL 61825.

Received 15 Mar. 2016; and in revised form 15 June 2016; accepted 16 July 2016 .
}

either from physical exertion or from environmental exposure. It results from direct thermal tissue injury as the core body temperature rises. Symptoms include hyperthermia, defined as a core body temperature of $40.5^{\circ} \mathrm{C}$ or greater, along with hot, dry skin, and central nervous system dysfunction. These symptoms may be preceded by nausea, vomiting, and muscle cramps. As the body temperature continues to rise above $42.4^{\circ} \mathrm{C}$, there is vasodilation with eventual circulatory collapse and cardiac failure (4).

Hyperthermia as a cause of death in the pediatric population is frequently highlighted by the lay press in the summer months. The overwhelming majority of these cases concern a young child who is left in a hot car by his or her caretaker. A review of the English literature regarding hyperthermia in children excluding natural disease and medication-associated causes ("hyperthermia NOT malignant NOT fever"-humans, English, child-birth to 18 years) yielded over 750 papers, most of which are similar to cases like these. For example, Krous et al. described ten cases of environmental hyperthermic infant and early childhood deaths. Eight of these deaths occurred in unattended vehicles, while two occurred in beds (5).

The authors encountered a fatal case of hyperthermia and thermal injuries resulting from proximate placement of a space heater in Detroit in January 2014. In addition, the scene was recreated using an analog to understand the progression of the findings observed in this heat-related death. These elements were integral parts of the investigation, as they were essential in the determination of manner of death.

\section{Case Summary}

A 16-month-old boy was found unresponsive in a stroller at home in Detroit in mid-winter. He was in the care of family 
members who were living in a house that was heated only by space heaters. The child, clad in a diaper and pants, was strapped in a stroller that was placed in front of a functioning EdenPURE space heater; a blanket was placed over the stroller and space heater, forming a tent. Reportedly, he was found unresponsive approximately $1 \mathrm{~h}$ after the time he was last known alive. When he was discovered, emergency medical services were contacted and he was conveyed to the hospital, where he was pronounced dead. Of note, a rectal temperature of $42.2^{\circ} \mathrm{C}$ was obtained on arrival to the hospital.

At autopsy, the child had first-degree burns on his face, chest, abdomen, arms with sparing of the palms and antecubital fossae, and right leg with sparing of the posterior thigh. There were blisters consistent with second-degree burns on the head, chest, abdomen, right flank, arms, and posterior right lower leg; several of the ruptured blisters had moist, erythematous bases and were consistent with antemortem injuries. Otherwise, there were no significant injuries or diseases. Postmortem toxicology was noncontributory.

\section{Materials and Methods}

To understand the time-course of the hyperthermia and to determine the progression of injuries to this child, the stroller, space heater, and blanket that were discovered at the scene of this child's death were acquired from the Detroit Police Department. A whole, freshly slaughtered porcine analog was obtained, which was slaughtered in accordance with USDA regulation and chosen to approximate the weight (25 pounds) and length (24 inches) of the child (Fig. 1). The analog had not been previously frozen and the internal organs had been removed. The analog was first heated in a hot water bath to simulate body temperature as closely as possible (Fig. 2). Using the specific heat of water $\left(4.19 \mathrm{~kJ} / \mathrm{kg}{ }^{\circ} \mathrm{C}\right)$ and an average of the published specific heats for pork products $\left(2.61 \mathrm{~kJ} / \mathrm{kg}{ }^{\circ} \mathrm{C}\right)$, it was calculated that it would take approximately $4 \mathrm{~h}$ and $48 \mathrm{~min}$ to raise the temperature of 55 gallons of water plus the analog from $21.1^{\circ} \mathrm{C}$ to $37^{\circ} \mathrm{C}(6,7)$.

Guided by the scene recreation photographs and notes, the heated analog was placed in the stroller; the space heater was positioned facing the stroller. The settings on the space heater were adjusted to match those that were noted at the time the child was found unresponsive. The blanket was draped over stroller and space heater, creating a "tent" (Fig. 3). Three temperature probes were used; one probe measured the temperature beneath the blanket within the "tent" (Plano thermometer), one measured the external temperature of the analog near the head (Klein Tools thermometer), and one measured the core temperature of the analog deep within its shoulder muscle (WilliamsSonoma). The external surface of the porcine analog was observed using an infant video monitor.

Temperatures from all three probes were recorded at the start of the experiment and at 15-min intervals. A visual inspection was also performed at these times using the infant video monitor. Photographs were taken at zero, 120, 180, and $240 \mathrm{~min}$ to record the condition of the analog's skin. When obtaining photographs, care was taken to retain the heat within the tent.

\section{Results}

The temperatures recorded by the three probes over four hours are plotted in Fig. 4. The temperature beneath the tent increased rapidly over the first $15 \mathrm{~min}$ by $37.7^{\circ} \mathrm{C}$ and then steadily by an average of $2.8^{\circ} \mathrm{C}$ per hour over the final $3 \mathrm{~h}$. The external temperature of the analog also increased rapidly over the first $15 \mathrm{~min}$ by $12.7^{\circ} \mathrm{C}$ and then steadily by an average of $1.1^{\circ} \mathrm{C}$ per hour over the final $3 \mathrm{~h}$. The core temperature of the analog steadily increased at an average of $3.2^{\circ} \mathrm{C}$ per hour throughout the entire experiment.

The condition of the analog's skin at $0 \mathrm{~h}, 2 \mathrm{~h}$, and $4 \mathrm{~h}$ is depicted in Fig. 5. The skin showed initial evidence of tanning at $105 \mathrm{~min}$. This discoloration darkened over the course of the experiment. No evidence of skin blisters or splits was observed.

\section{Discussion}

To further comprehend situations in which hyperthermia can occur, one must first understand the fundamentals of heat transfer. There are three modes by which heat is transferred: conduction, convection, and radiation (5). Conduction occurs when there is a temperature gradient in a stationary medium, whether that medium is a solid or fluid. It can be thought of as transfer of energy from the more energetic to the less energetic particles

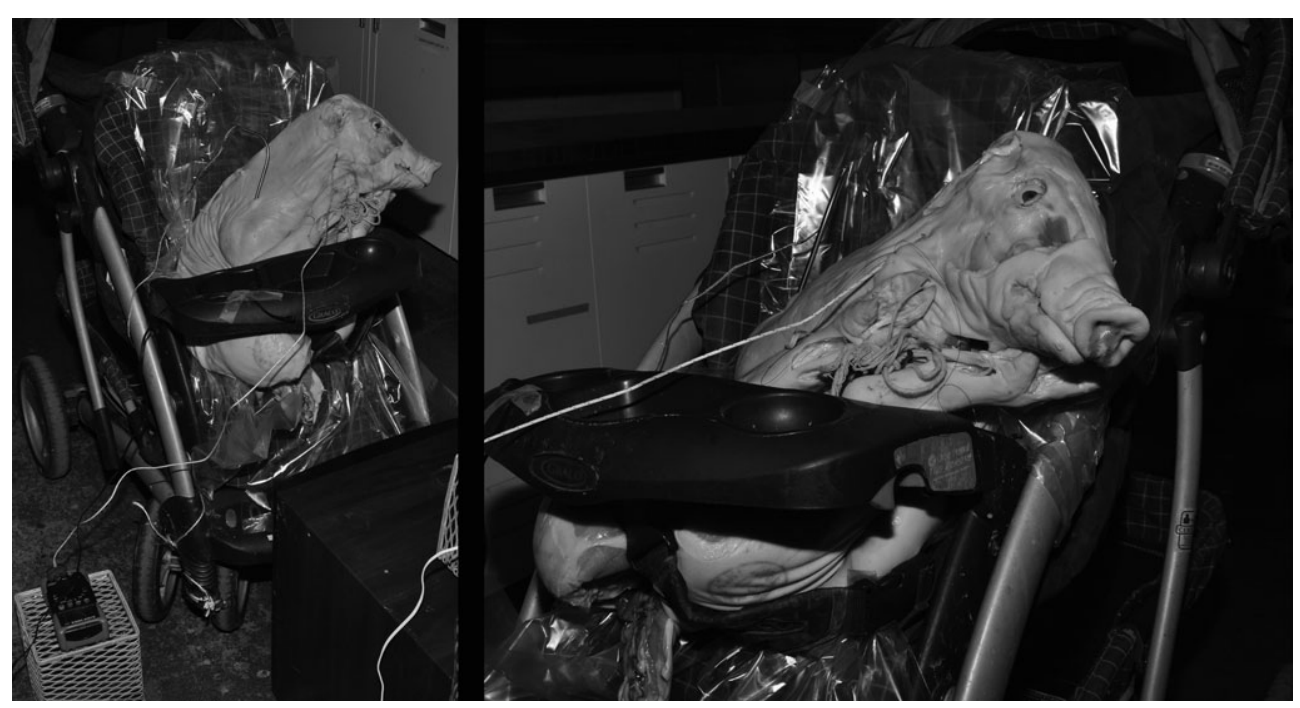

FIG. 1-Porcine analog. 


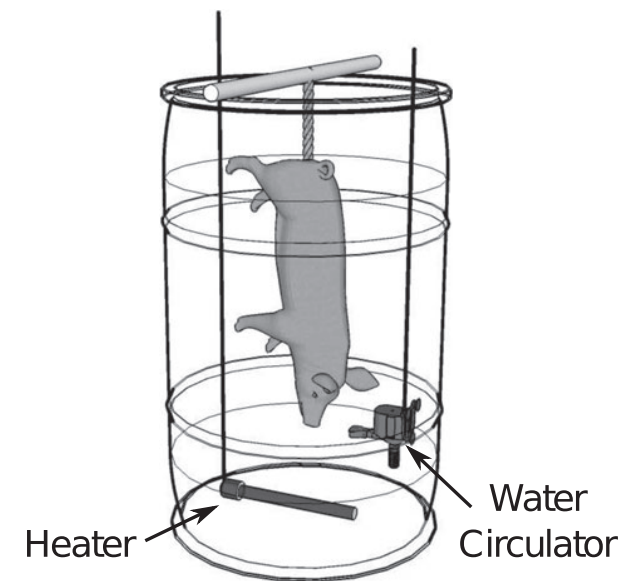

FIG. 2-Schematic diagram of the method used to warm the porcine analog to human body temperature.

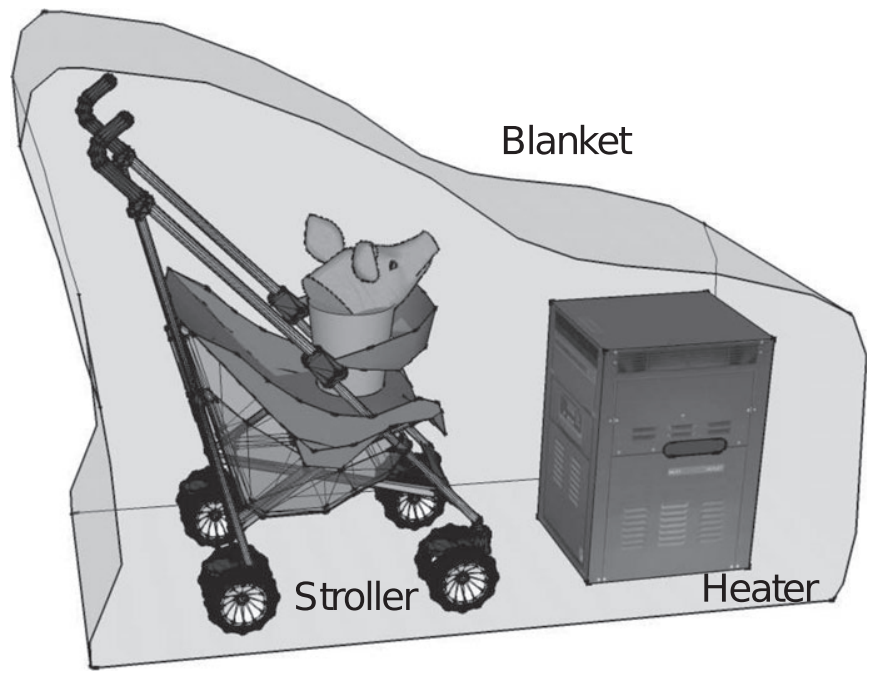

FIG. 3-Schematic diagram of the scene recreation.

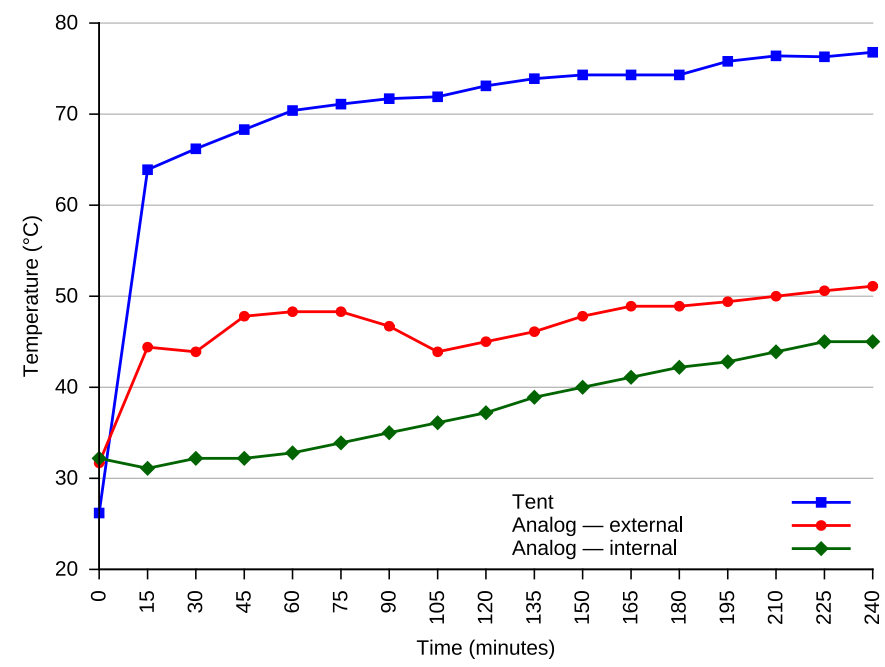

FIG. 4-Temperatures within the tent and of the porcine analog, recorded at 15-min intervals. [Color figure can be viewed at wileyonlinelibrary.com] of a substance due to the interactions among those particles (5). Convection refers to heat transfer between a surface and a moving fluid in the presence of a temperature gradient. It is composed of two mechanisms: diffusion and the bulk motion of the fluid. Moreover, there are several types of convection, including forced convection and free (natural) convection. Forced convection occurs when the flow is caused by an external means, such as a fan or a pump. In free convection, the flow is caused by buoyancy forces that induce motion (5). Radiation is energy that is emitted by matter that is at a finite temperature. It does not require the presence of a medium material and occurs most efficiently in a vacuum (5).

For obvious ethical reasons, pediatric hyperthermia-related deaths have not been explored experimentally. In this case, it was essential to understand the progression of findings and timecourse of the hyperthermia, as this could alter the manner of death from accident to homicide. Therefore, the literature was searched to find cases that were analogous to this one to understand the progression of findings that can be seen in these deaths. The most commonly encountered scenario involves a child who is left in a vehicle, either accidently or intentionally. In these cases, the primary modes of heat transfer are conduction and natural convection. Radiation is also involved, as it provides the heat to the system $(6,8)$. Booth et al. discussed 231 hyperthermia deaths from 1999 to 2007 among children in parked vehicles (9). These authors noted that the amount of time between when the decedent was last known alive and when he/ she was found dead or dying averaged $4.6 \mathrm{~h}$ and ranged from 0.25 to $16 \mathrm{~h}$ (9). They referred to the paper by McLauren et al., wherein the investigators noted that the temperature within an enclosed vehicle rises on average $3.2^{\circ} \mathrm{F}$ per $5 \mathrm{~min}$ (10). Eighty percent of the total temperature increase occurred within the first 30 min. Maximum temperature occurred at approximately $60 \mathrm{~min}$ and was on average $41^{\circ} \mathrm{F}$ higher than the starting ambient temperature. Moreover, a comparison of closed versus "cracked" windows showed no significant difference in rate of temperature increase or final temperature (10).

An interesting study was performed by Grunstein et al. in which they used a human heat balance model to simulate a male infant's physiological response to extreme heat within an enclosed vehicle (11). They performed this observational study in an enclosed vehicle 1 day per month and collected data from 8:00 am until 4:00 pm, recording the time to un-compensable heating, heat stroke, and critical thermal maximum. In all four seasons, despite differences in starting temperature and solar radiation, the model infant reached heat stroke and demise before 2:00 pm (11).

Another scenario similar to that of the vehicle-associated cases occurs in a sauna, where the heat transfer modes include both conduction and natural convection. Saunas are large enclosures generally without ventilation that are heated by electricity, gas, or other means and are maintained at temperatures of $70-90^{\circ} \mathrm{C}$ (12). Press studied the health hazards of saunas and spas (12). He surveyed medical examiners and coroners and collected a series of seven fatalities that occurred in saunas. Notably, all of these individuals were 12 years of age or older. Six of the seven decedents had risk factors that have been reported to be associated with spa and sauna deaths, including alcohol intoxication or heart disease (12).

The manufacturer of the EdenPURE space heater found at the scene and used in this experiment states that these heaters use infrared bulbs and copper heating chambers that warm the air that is forced out into an area (13). Thus, in this case and in 


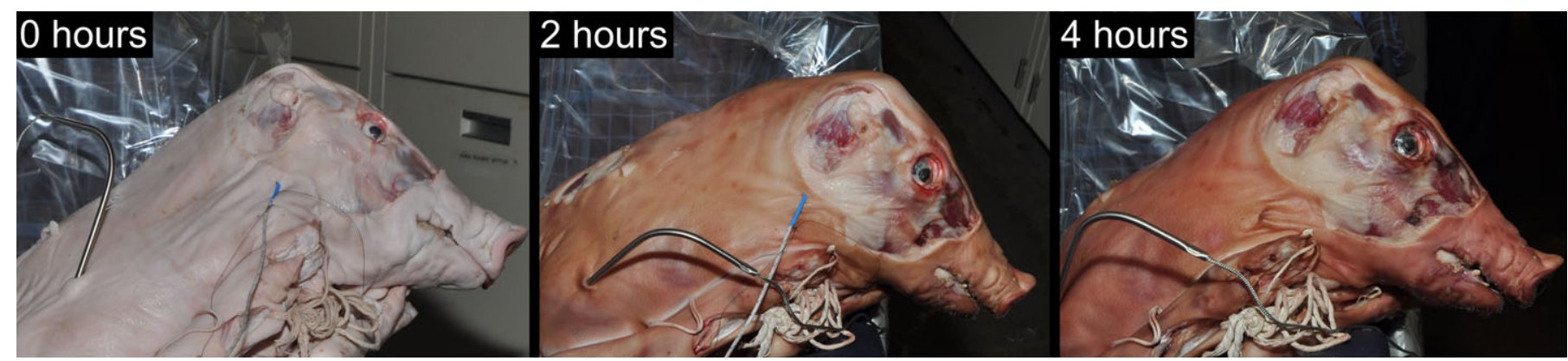

FIG. 5-Progression of the skin condition of the porcine analog over the duration of the experiment. [Color figure can be viewed at wileyonlinelibrary.com]

vehicles that are heated by the sun, conduction and convection occur. However, in this case, the convective heat transfer was a result of forced convection. The way in which the heat was delivered to the system also differed. To best understand the situation that occurred in this case, an attempt was made to replicate the scenario using a porcine analog. The following strategy was used to first search the English literature for nonhuman hyperthermia experiments using porcine analogs: (pig OR swine OR porcine) AND hyperthermia NOT fever NOT malignant. There were approximately 430 articles, most of which were treatment-related and imaging studies. This current experimental model and arrangement has not yet been reported.

In this case, burns were also noted on the child, some of which were on "nonexposed," posterior aspects of the child's limb surfaces. Burns are generally divided into six categories: flame, contact, radiant, scalding, microwave, and chemical burns. The severity of the burn is dependent upon the intensity of the heat and the duration of exposure (4). In the 1940s, Mortiz et al. performed experiments on volunteers to determine the timesurface temperature thresholds for thermal injury of human skin (14). They found that skin heated to $45^{\circ} \mathrm{C}$ for $2 \mathrm{~h}$ produced first-degree burns, while $3 \mathrm{~h}$ of exposure produced complete epidermal necrosis (14). In the study by Krous et al. in which they described ten cases of environmental hyperthermic infant and early childhood deaths, the most common finding on external examination was evidence of dehydration. However, two of the children discovered in vehicles showed skin slippage; one of these children who had an exposure time of $3.5 \mathrm{~h}$ also had cutaneous burns (5). In this current experiment, tanning of the porcine skin was noted, a surrogate marker of burning, at $105 \mathrm{~min}$, when the external skin surface of the analog was $43.9^{\circ} \mathrm{C}$. This correlates well with what was observed in Mortiz's study.

There are limitations to this experimental model. The starting temperature of the analog was lower than that of normal body temperature. Therefore, it would have taken longer for the ana$\log$ to reach the temperature that the child reached in this case, making the neglect element of the scenario potentially more problematic to prove. In addition, the analog did not auto-regulate its temperature as a human would have in the early stages of the experiment. The recorded temperatures would have been even higher had the analog started at body temperature and had it retained heat at the start of the experiment. Tanning was used as a surrogate marker of thermal burns. However, this is not a perfect simulation, as the analog's skin would not have responded like a living human's skin (i.e., no innervation, no vascular supply).

From the literature searches and this experimental model, it was determined that the child was left strapped in the stroller facing the space heater for longer than the reported $1 \mathrm{~h}$ between the time he was last known alive and found unresponsive. Using tanning of the analog's skin as a surrogate marker of skin burns, it was clear that surface damage occurred closer to $2 \mathrm{~h}$ after the heating began. Burns of the "nonexposed," posterior aspects of the child's limb surfaces were interpreted as an indication of pain and struggle. Therefore, it is the authors' opinion that neglect contributed to this child's death and the manner was certified as homicide.

\section{Conclusions}

The authors encountered an unusual pediatric heat-related death due to a space heater. The scenario was replicated using a whole, freshly slaughtered porcine analog. This experimental model was essential to the investigation into cause and manner of death because it demonstrated the length of time required to produce these injuries. The cause of death was hyperthermia and thermal burns, with neglect as a contributing factor. The manner of death was homicide.

\section{References}

1. Donoghue ER, Graham MA, Jentzen JM, Lifschultz BD, Luke JL, Mirchandani HG. Criteria for the diagnosis of heat-related deaths: National Association of Medical Examiners. Position paper. National Association of Medical Examiners Ad Hoc Committee on the Definition of HeatRelated Fatalities. Am J Forensic Med Pathol 1997;18(1):11-4.

2. https://www.nlm.nih.gov/medlineplus/heatillness.html (accessed March 13, 2016).

3. Lugo-Amador NM, Rothenhaus T, Moyer P. Heat-related illness. Emerg Med Clin North Am 2004;22(2):315-27, viii.

4. DiMaio VJM, Dana SE. Handbook of forensic pathology, 2nd edn. Boca Raton, FL: CRC Press, Taylor \& Francis Group, 2007.

5. Krous HF, Nadeau JM, Fukumoto RI, Blackbourne BD, Byard RW. Environmental hyperthermic infant and early childhood death: circumstances, pathologic changes, and manner of death. Am J Forensic Med Pathol 2001;22:374-82.

6. Incropera FP, DeWitt DP. Introduction to heat transfer, 4th edn. New York, NY: John Wiley \& Sons Inc, 2002.

7. http://www.engineeringtoolbox.com/specific-heat-capacity-foodd_295.html (accessed March 13, 2016).

8. Grundstein AJ, Dowd J, Meentemeyer V. Quantifying the heat-related hazard for children in motor vehicles. Bull Am Meteorol Soc 2010;91:1183-91.

9. Booth JN 3rd, Davis GG, Waterbor J, McGwin G Jr. Hyperthermia deaths among children in parked vehicles: an analysis of 231 fatalities in the United States, 1999-2007. Forensic Sci Med Pathol 2010;6(2):99-105.

10. McLaren C, Null J, Quinn J. Heat stress from enclosed vehicles: moderate ambient temperatures cause significant temperature rise in enclosed vehicles. Pediatrics 2005;116(1):109-12.

11. Grundstein AJ, Duzinski SV, Dolinak D, Null J, Iyer SS. Evaluating infant core temperature response in a hot car using a heat balance model. Forensic Sci Med Pathol 2015;11(1):13-9. 
12. Press E. The health hazards of saunas and spas and how to minimize them. Am J Public Health 1991;81(8):1034-7.

13. http://www.edenpure.com/edenpure-coppersmart-1000-portable-infraredheater (accessed March 13, 2016).

14. Moritz AR, Henriques FC. Studies of thermal injury: II. The relative importance of time and surface temperature in the causation of cutaneous burns. Am J Pathol 1947;23(5):695-720.
Additional information and reprint requests:

Amanda O. Fisher-Hubbard, M.D.

Western Michigan University Homer Stryker M.D. School of Medicine 1000 Oakland Drive

Kalamazoo, MI 49008

E-mail: amanda.fisher-hubbard@med.wmich.edu 